\title{
Severe head injury in elderly: 6-year comparison of treatment and outcome between southern Finland and Navarra (Spain)
}

\author{
Bismil Ali Ali ${ }^{1}$ Tuomas Brinck ${ }^{2} \cdot$ Lauri Handolin $^{2} \cdot$ Tomas Belzunegui Otano $^{1,3}$
}

Received: 13 September 2018 / Accepted: 7 February 2019 / Published online: 12 February 2019

○) Springer-Verlag GmbH Germany, part of Springer Nature 2019

\begin{abstract}
Purpose To compare the profile, treatment and outcome of elderly patients with severe traumatic brain injuries (TBI) between southern Finland and Navarra (Spain).

Methods Data collected from, 2010 to 2015, in the Major Trauma Registry of Navarra (MTR-N) and the Helsinki Trauma Registry (HTR) were compared. Patients with New Injury Severity Score (NISS) $\geq 16$ and age $\geq 65$ with isolated severe TBI were considered. Patients who had been admitted to the hospital $\geq 24 \mathrm{~h}$ after the trauma, had been pronounced dead before hospital arrival, or had been injured by hanging, drowning or burns, were excluded. Outcome was defined by 30 -day hospital mortality. The expected mortality was calculated using the Revised Injury Severity Classification score II (RISC II). Other compared data included demographics, injury mechanism, pre-hospital and hospital treatment, and time intervals. Results A total of 305 (MTR-N) and 137 (HTR) patients were included in the outcome analysis. The standardized mortality ratio with 95\% confidence interval was for MTR-N 1.4 (1.1-1.6) and for HTR $0.8(0.6-1.1)$. Patients in Navarra were older (average 79.7 vs. 75.0 ) while in southern Finland the percentage of pre-hospital intubation in patients with GCS $\leq 8$ (75.0\% vs $50.0 \%)$ and ICU admission ( $72.2 \%$ vs $22.0 \%$ ) were higher.

Conclusion The better adjusted outcome of elderly patients with severe TBI in southern Finland in comparison to Navarra could be due to higher rate of pre-hospital intubation and/or higher rate of ICU admissions in southern Finland. Increasing number of elderly patients with severe TBI necessitate uniformly accepted protocols in pre- and in-hospital management.
\end{abstract}

Keywords Elderly trauma patients $\cdot$ Severe traumatic brain injury $\cdot$ Major trauma $\cdot$ Trauma registry $\cdot$ Benchmarking

\section{Introduction}

Major trauma (MT) is a worldwide pandemic and it can be defined using a New Injury Severity Score (NISS) threshold of 15 [1]. Along with increasing, more active and longerliving elderly people, MT in the geriatric population is becoming increasingly recognized as a significant challenge to health systems $[2,3]$. The literature usually tends to define old age in trauma as corresponding to 65 years and above

Bismil Ali Ali

bismilnawazish@gmail.com

1 Accident and Emergency Department, Complejo Hospitalario de Navarra, Health service of NavarraOsasunbidea, 31011 Pamplona, Spain

2 Trauma Unit, Helsinki University Hospital, University of Helsinki, Helsinki, Finland

3 Department of Health, Public University of Navarra, Pamplona, Spain
[4]. As mean population age increases globally, the proportion of people aged 65 and older in Europe is expected to grow to at least $30 \%$ by 2050 [5].

Several studies worldwide have found higher mortality rates in older trauma patients compared to younger adults with similar injuries [6, 7]. Lower impact mechanisms of injury, such as falls, can produce serious, life-threatening injuries [8]. Likelihood of falls increases with age associated with multiple factors (such as hearing problems, poor vision, muscle weakness, slow reactions, cognitive impairment and previous disabilities) as does the probability of significant injury as a consequence, while physiological reserve and the ability to recover is diminished [9].

Fall in the elderly may lead to a severe brain injury-the leading cause of injury deaths and disabilities among persons aged $>65$ years [10]. These patients are commonly using systemic anticoagulants which can add intracranial bleeding [11]. Rehabilitation of geriatric patients with traumatic brain injury (TBI) is challenging, and full recovery 
can be difficult leading to loss of independence. Elderly trauma patients with severe head injury pose an increasing challenge for healthcare and economics [12]. Uniformly accepted protocols in both pre- and in-hospital management as well as rehabilitation are lacking.

The aim of this study was first to compare incidence, demographics, injury profile, treatment and outcome of elderly patients with severe head trauma between southern Finland and Navarra using their corresponding trauma registries. Secondly, an outcome analysis was performed, to find out possible differences in the quality of care between southern Finland and Navarra.

\section{Materials and methods}

\section{Study populations}

We compared the data from the Major Trauma Registry of Navarra (MTR-N) to that of the Helsinki Trauma Registry (HTR) in a period from 1.1.2010 until 31.12.2015. Patients with (NISS) $\geq 16$ and age $\geq 65$ with isolated severe head injuries (defined as head abbreviated injury scale (AIS) $\geq 3$, no other AIS $>2$ [13]) were considered. Patients who had been admitted to the hospital $\geq 24 \mathrm{~h}$ after the trauma, who were dead on arrival (defined as arriving at hospital with no signs of life), who had been injured by hanging, or drowning or burnt patients, were excluded. Furthermore, we excluded patients transferred from another hospital due to missing initial status on admission and primary admitted patients transferred to another hospital after initial treatment (within $48 \mathrm{~h}$ ) due to unknown outcome.

\section{Trauma system in Navarra and southern Finland}

The trauma system of Navarra is publicly funded, and it gives coverage to an area of $10,421 \mathrm{~km}^{2}$ and a population of 637,000 inhabitants. It is divided into three areas: Pamplona, Tudela and Estella. Complejo Hospitalario de Navarra (CHN) in Pamplona constitutes the only tertiary referral hospital. When necessary, two local hospitals (Hospital Reina Sofia de Tudela and Hospital Garcia Orcoyen de Estella) can provide initial trauma care while awaiting the proper timing for transportation to the CHN. Neurosurgical treatment is performed in CHN. Thus, patients with known TBI or suspected to have sustained head injury are directly transferred to CHN [14].

The pre-hospital management in both regions is coordinated by a center, which mobilizes resources for outpatient care (physicians or paramedics) according to the seriousness of the victim's condition. In Pamplona, there are two physician-staffed ambulances that provide medical assistance to the whole area. The areas of Tudela and
Estella each have one physician-staffed ambulance at their hospitals [14]. The physicians working prehospital setting and in hospital are usually specialized in family medicine with post-graduate emergency medicine training. The annual number of trauma patients in Navarra with NISS $\geq 16$ is about 200 .

In southern Finland the treatment of severe blunt injuries of adult patients ( $>16$ years) is centralized to the trauma unit of Helsinki University Hospital (HUH) with a catchment area of 1.8 million people $(25 \%$ of the total Finnish population) and a range up to $200 \mathrm{~km}$. Annual number of NISS $\geq 16$ trauma patients in the HUH trauma unit is around 440. Hospitals treating major trauma patients in Finland are publicly funded. Traffic accidents and work-related accidents are covered by law-enforced insurance. In southern Finland there are ambulance doctor and helicopter doctor services with good coverage.

The doctors working pre-hospital are usually anesthesiologists specialized in emergency care. The HUH trauma unit is the only hospital with neurosurgical capacity in the area. The field triage of the pre-hospital system dictates all the suspected severe head trauma to be transferred directly to the HUH trauma unit.

In both Navarra and southern Finland, trauma care is performed according to the Advanced Trauma Life Support [15] or European Trauma Course (ETC) Guidelines [16].

\section{The registries: MTR-N and HTR}

In Finland and Spain there are no strict national guidelines for pre- or intra-hospital care of trauma patients, nor any nation-wide trauma registry.

The MTR-N was founded in 2010 with the aim of internal and external benchmarking. A specific Web application, which enables the cooperation of various users in data collection, was developed to register patients. The users of this application are all doctors from the hospital and pre-hospital emergency care departments and those of the intensive care units of the public health system of Navarra, with an approximate total of 150 users. A data manager is responsible for the general supervision and administration of the system, as well as for verifying the compliance of the inclusion criteria and of the introduction of patient data [17].

The HTR was established in 2005 as a benchmarking project to improve regional trauma-patient outcome. Five dedicated trauma nurses enter the data into the HTR [18]. Only patients with NISS $\geq 16$ treated in the resuscitation room of HUH trauma center are included.

Both MTR-N and HTR follow the Utstein template for uniform reporting of data after major trauma [1]. 


\section{Comparisons}

The following parameters were compared between MTR-N and HTR: age, sex, pre-injury ASA, injury scoring (ISS and NISS), injury pattern, mechanism of injury, intention of injury, pre-hospital timings, transportation method, prehospital intubation, treatment at hospital, discharge destination and 30-day in-hospital mortality.

All parameters from MTR-N and HTR were checked for comparability, and for some variables transformations had to be done before the analysis. All comparisons are based on real measurements; no imputations for patients with missing data were performed. The statistical analysis was performed with the Statistical Package for the Social Sciences (SPSS version 23, IBM Inc., Armonk NY, USA).

\section{Outcome analysis}

We defined 30-day in-hospital mortality as death in reporting hospital within 30 days from admission. For reason of comparability, patients who died beyond day 30 were considered survivors in this analysis.

Expected mortality was defined as the average value of individual prognoses derived from the Revised Injury Severity Classification score II (RISC II) [19]. Standardized mortality ratio (SMR) was defined as the ratio of observed and expected mortality.

\section{Ethics approval}

This study received approval from the local medical ethics committee of Navarra, Spain and was approved by the administrative board of the HTR.

\section{Results}

\section{Patient characteristics}

We included 305 patients from MTR-N and 137 patients from HTR (Fig. 1). The characteristics of the included patients are shown in Table 1 . The average age was $79.7 \pm 7.7$ in Navarra and $75.0 \pm 7.4$ in HTR. The average NISS in Navarra and southern Finland was $26.2 \pm 8.6$ and $32.0 \pm 10.9$, respectively. The share of head-AIS $5 / 6$ patients was higher in HTR (59.9\% vs. $36.1 \%)$.

Blunt injury mechanism was more prominent in Navarra than in southern Finland (99.7\% vs. 91.2\%). Information on type, intention and mechanism of injury is included in Table 2. Accidental intention was more frequent in Navarra

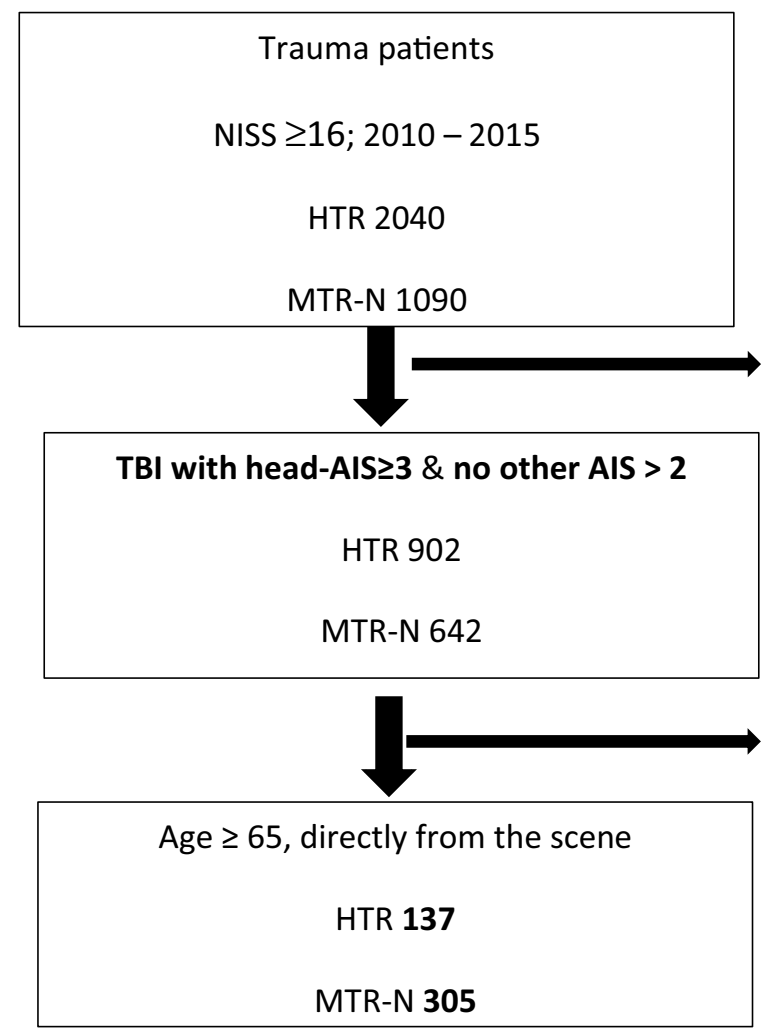

Excluded

TBI with head-AIS $<3$ \&/or AIS $>2$ in other regions

HTR 1139

MTR-N 448

\section{Excluded \\ - $\quad$ age $<65$ \\ HTR 562 \\ MTR-N 325 \\ - transfers \\ HTR 202 \\ MTR-N 12}

Fig. 1 Flow diagram of included and excluded patients in outcome analysis. NISS New injury severity score, MTR-N Major Trauma Registry of Navarra, HTR Helsinki Trauma Registry, TBI traumatic brain injury, AIS Abbreviated Injury Scale 
Table 1 Characteristics of patients

\begin{tabular}{lll}
\hline & MTR-N & HTR \\
\hline$N$ & 305 & 137 \\
Age, mean (SD) median (M) & $79.7 \pm 7.7$ & $75.0 \pm 7.4$ \\
& M: 80 & M: 73 \\
Male gender & $160(52.5 \%)$ & $91(66.4 \%)$ \\
Pre-injury ASA-PS classification system & & \\
Normal healthy patients (1) & $63(20.7 \%)$ & $23(16.8 \%)$ \\
Mild systemic disease (2) & $189(62.0 \%)$ & $66(48.2 \%)$ \\
Severe systemic disease (3 or 4) & $53(17.4 \%)$ & $48(35.0 \%)$ \\
ISS, mean (SD) median (M) & $19.7 \pm 6.7$ & $22.1 \pm 6.1$ \\
& M:16 & M:25 \\
NISS, mean (SD) median (M) & $26.2 \pm 8.6$ & $32.0 \pm 10.9$ \\
Head AIS 5 & M: 25 & M: 29 \\
Head AIS 6 & $109(35.5 \%)$ & $82(59.9 \%)$ \\
\hline
\end{tabular}

Table 2 Type, intention and mechanism of injury

\begin{tabular}{lcc}
\hline & MTR-N & HTR \\
\hline Type of injury & & \\
Blunt & $304(99.7 \%)$ & $125(91.2 \%)$ \\
Intention & & \\
Accidental & $290(95.1 \%)$ & $105(76.6 \%)$ \\
Assault & $3(1.0 \%)$ & $3(2.2 \%)$ \\
Self-inflicted & $2(0.7 \%)$ & $8(5.8 \%)$ \\
Unknown & $10(3.3 \%)$ & $21(15.3 \%)$ \\
Mechanism of injury & & \\
Traffic (motor vehicle, motorcy- & $44(14.4 \%)$ & $21(15.3 \%)$ \\
$\quad$ cle, bicycle, pedestrian) & & \\
Gunshot wounds or stabbing & $1(0.3 \%)$ & $5(3.6 \%)$ \\
Hit by blunt object & $2(0.7 \%)$ & $1(0.7 \%)$ \\
Low fall $(<3$ m) & $233(76.4 \%)$ & $74(54.0 \%)$ \\
High fall (>3 m) & $17(5.6 \%)$ & $11(8.0 \%)$ \\
Others & $4(1.3 \%)$ & $5(3.6 \%)$ \\
Unknown & $4(1.3 \%)$ & $20(14.6 \%)$ \\
\hline
\end{tabular}

(95.0\% vs. $76.7 \%)$ while self-inflicted injuries were more common in southern Finland (5.8\% vs. $0.6 \%$ ).

Falls from low height as an injury mechanism were more usual in MTR-N compared to HTR (76.3\% vs. $54.0 \%)$.

\section{Pre-hospital setting}

Pre-hospital details regarding physiology, treatment, transportation method and time intervals are shown in Table 3. In southern Finland, patient was more commonly treated by a doctor at pre-hospital settings than in Navarra (50.4\% vs. $46.8 \%$, respectively). More unconscious patients were documented in HTR (41.3\%) than in Navarra (16.4\%). Prehospital intubation rates were higher in southern Finland
Table 3 Pre-hospital data

\begin{tabular}{lll}
\hline & MTR-N & HTR \\
\hline Systolic blood pressure & $142.1 \pm 23.1$ & $160.9 \pm 32.2$ \\
& M: 140 & M: 160 \\
Glasgow Coma Scale (GCS) & $12.5 \pm 3.8$ & $8.4 \pm 4.7$ \\
& M: 15 & M: 8 \\
Intubation (all) & $66(21.6 \%)$ & $59(43.1 \%)$ \\
A supraglottic device airway (all) & $38(12.5 \%)$ & $0(0 \%)$ \\
Unconscious (GCS $\leq 8)$ & $50(16.4 \%)$ & $72(52.6 \%)$ \\
Intubation (GCS $\leq 8)$ & $25(50.0 \%)$ & $54(75.0 \%)$ \\
A supraglottic device airway (GCS $\leq 8)$ & $3(6.0 \%)$ & $0(0 \%)$ \\
Cardio-pulmonary resuscitation & $2(0.7 \%)$ & $1(0.7 \%)$ \\
Pre-hospital fluids given, $n(\%)$ & $190(62.3 \%)$ & $127(92.7 \%)$ \\
Amount of volume, if given (ml) & $444 \pm 409$ & $725 \pm 500$ \\
& M: 500 & M: 500 \\
Transport method & & \\
Ambulance & $278(91.1 \%)$ & $132(96.4 \%)$ \\
Helicopter ambulance & $6(2.0 \%)$ & $2(1.5 \%)$ \\
Private vehicle & $20(7.0 \%)$ & $1(0.7 \%)$ \\
Others & $0(0 \%)$ & $2(1.5 \%)$ \\
Doctor on scene & $143(46.9 \%)$ & $69(50.4 \%)$ \\
Pre-hospital timings & & \\
Time from alarm to arrival at scene & $0: 19 \pm 0: 12$ & $0: 14 \pm 0: 08$ \\
On scene time & M: 15 & M: 10 \\
Time from alarm to hospital & $0: 34 \pm 0: 21$ & $0: 36 \pm 0: 23$ \\
& M: 30 & M: 35 \\
& $1: 06 \pm 0: 33$ & $1: 12 \pm 0: 34$ \\
& M: $1: 01$ & M: $1: 11$ \\
\hline & & \\
& &
\end{tabular}

than in Navarra (43.1\% vs. $21.6 \%$, respectively). In patients with first measured GCS $\leq 8$, (MTR-N 50 out of 305, 16.4\%; HTR 72 out of 137, 52.6\%) the pre-hospital intubation rate in Navarra was $25(50.0 \%)$ and in southern Finland 54 $(75.0 \%)$.

In pre-hospital setting, patients in southern Finland receive more volume than in Navarra $(88.6 \%$ with a median of $1000 \mathrm{ml}$ vs. $62.3 \%$ with a median of $500 \mathrm{ml}$ ). Regarding response times, Navarra's pre-hospital team arrive later than the pre-hospital team in southern Finland (0:19 $\pm 0: 12$ vs. 0:14 $\pm 0: 08)$ but in southern Finnish team few more time is spent on scene than in Navarra (0:36 $\pm 0: 23$ vs. $0: 34 \pm 0: 21)$.

\section{Diagnostic procedures and treatment at hospitals}

More head CT scans were performed in Navarra's hospitals than in the HUH trauma unit (100\% vs. $97.1 \%$, respectively). It took more time to perform first CT scan and first surgical intervention after admission in Navarra than in Helsinki (0:40 and 1:52 vs. $0: 23$ and 1:23, respectively). Table 4 illustrates information related to the diagnostic procedures and treatment at hospital. 
Table 4 Hospital data and outcomes of severely injured patients of both regions

\begin{tabular}{|c|c|c|}
\hline & MTR-N & HTR \\
\hline Arterial base excess & $\begin{array}{l}-3.1 \pm 4.0 \\
\mathrm{M}:-2.0\end{array}$ & $\begin{array}{l}-2.7 \pm 3.7 \\
\mathrm{M}:-2.3\end{array}$ \\
\hline Coagulation: INR & $\begin{array}{l}1.4 \pm 0.8 \\
\mathrm{M}: 1.0\end{array}$ & $\begin{array}{l}3.2 \pm 2.2 \\
\text { M: } 2.9\end{array}$ \\
\hline Systolic BP & $\begin{array}{l}145 \pm 24 \\
\text { M: } 144\end{array}$ & $\begin{array}{l}156 \pm 35 \\
\text { M: } 160\end{array}$ \\
\hline GCS on admission & $11.8 \pm 3.4$ & $9.8 \pm 4.6$ \\
\hline Head computed tomography (CT) performed (\%) & $305(100 \%)$ & $133(97.1 \%)$ \\
\hline Time to first CT scan & $\begin{array}{l}0: 43 \pm 0: 23 \\
\text { M: 0:40 }\end{array}$ & $\begin{array}{l}0: 27 \pm 0: 16 \\
\text { M: 0:22 }\end{array}$ \\
\hline ICU treatment $(\%)$ & $66(22.0 \%)$ & $99(72.2 \%)$ \\
\hline Ventilated $(\%)$ & $42(14.0 \%)$ & $77(56.2 \%)$ \\
\hline Ventilation days (if ventilated) & $\begin{array}{l}4.6 \pm 7.7 \\
\text { M: } 1\end{array}$ & $\begin{array}{l}2.9 \pm 4.1 \\
\text { M: } 1\end{array}$ \\
\hline Length of hospital stay (days) & $\begin{array}{l}9.0 \pm 12.3 \\
\text { M: } 5\end{array}$ & $\begin{array}{l}9.0 \pm 10.0 \\
\text { M: } 6\end{array}$ \\
\hline 30 days in-hospital mortality & $110(36.0 \%)$ & $40(29.2 \%)$ \\
\hline \multicolumn{3}{|l|}{ Discharge destination (survivors only) } \\
\hline Home & $145(74.4 \%)$ & $10(10.3 \%)$ \\
\hline Rehabilitation & $12(6.2 \%)$ & $2(2.1 \%)$ \\
\hline Another critical care unit & $1(0.5 \%)$ & $7(7.2 \%)$ \\
\hline Another intermediate or low care somatic hospital ward & $36(18.5 \%)$ & $78(80.4 \%)$ \\
\hline Others & $1(0.5 \%)$ & \\
\hline \multicolumn{3}{|l|}{ Glasgow Outcome Scale at discharge } \\
\hline Good recovery & $153(78.5 \%)$ & $8(8.2 \%)$ \\
\hline Moderate disability & $25(12.8 \%)$ & $17(17.5 \%)$ \\
\hline Severe disability & $15(7.7 \%)$ & $57(58.8 \%)$ \\
\hline Persistent vegetative state & $2(1.0 \%)$ & $15(15.5 \%)$ \\
\hline 30-day in-hospital mortality & $110(36.1 \%)$ & $40(29.2 \%)$ \\
\hline Standardized mortality ratio $(95 \% \mathrm{CI})$ & $1.4(1.1-1.6)$ & $0.8(0.6-1.1)$ \\
\hline
\end{tabular}

Standardized mortality ratio is defined as the ratio of 30 days in-hospital mortality to expected mortality, which is the average value of individual prognoses derived from the Revised Injury Severity Classification Score II (RISC II)
The patients were more likely to be admitted to ICU in HUH than in Navarra's hospitals $(72.2 \%$ vs. $22.0 \%$, respectively). Higher percentage of ventilated patients ( $56.2 \%$ vs. $14.0 \%)$ were observed in HUH than in Navarra.

\section{Discharge destination and Glasgow Outcome Scale}

In Navarra, $74.3 \%$ (145 of 195) of survived patients were discharged home directly from the hospital, compared with $10.3 \%$ (10 of 97) in southern Finland. The number of patients discharged to another intermediate or low care somatic hospital ward was higher in southern Finland than in Navarra ( $80.4 \%$ vs. $18.5 \%$ ). Measured with the Glasgow Outcome Scale (GOS) at discharge, $58.8 \%$ of the survivors documented in HTR had severe disability compared to $7.7 \%$ of Navarra's patients.

\section{0 days in-hospital mortality and RISC II analysis}

The 30-day in-hospital mortality was higher in MTR-N than in HTR (36.0\% vs. $29.2 \%$ ). This difference was not statistically significant $(p=0.16)$. The expected mortality according to RISC II was $29.7 \%$ for MTR-N and $36.5 \%$ for HTR. The standardized mortality ratio (SMR, observed mortality divided by the expected morality) with $95 \%$ confidence interval is $1.4(1.1-1.6)$ for MTR-N and $0.8(0.6-1.1)$ for HTR.

\section{Discussion}

The outcome of elderly patients with severe head trauma differed clearly between southern Finland and Navarra. Both observed mortality and standardized mortality ratio (defined 
as the ratio of observed to expected mortality) indicated better results for southern Finland compared to Navarra.

This result might be due to the difference in the trauma care systems and/or treatment (more frequent pre-hospital intubation of unconscious patients and higher admission rate to ICU in southern Finland) and/or different features of the populations (older patients in Navarra).

It is well known that the mortality rate for patients aged $\geq 65$ years is significantly higher than in younger trauma patients [20]. In our study selected group of aged patients, Navarra's patients were still older than Finnish. This could explain partially the higher mortality found in Navarra. On the other hand, age is taken into consideration in expected mortality-calculations as defined by RISC II [19]: the higher the age, the worse the prognosis. Thus, the difference seen also in the standardized mortality ratio indicating better results in southern Finland is not explained by the differing age between the registries.

Pre-hospital intubation rates documented in HTR were higher than those recorded in MTR-N. This might partially explain the better outcome of patients treated in southern Finland. However, several studies have reported increased failure rates and severe complications in trauma patients who were intubated prehospital setting [21]. On the other hand, airway management with other adjuncts like laryngeal mask have proved useful in pre-hospital airway management [22]. In southern Finland, supraglottic devices are seldom used prehospital setting. In Navarra, supraglottic device was used in 38 cases (12.4\%). Furthermore, GCS $\leq 8$ is a general indication for intubation in southern Finland as well as in Navarra. In this study, MTR-N had lower intubation rates even in patients with GCS $\leq 8$. The high number of non-intubations for patients with initial GCS $\leq 8$ can be considered as a failure in the pre-hospital system. Intubation of unconscious patients should be given special attention when reorganizing pre-hospital care.

The average volume of given pre-hospital fluids was higher in southern Finland. In general, the pre-hospital fluids should be limited to a relatively small volume in trauma patients. The unnecessary fluid administration may lead to increased bleeding by raising the blood pressure, dilution of coagulation factors, cooling down of the patient, all of which can lead to coagulopathy. On the other hand, in patients with suspected brain injury the blood pressure should be kept high enough (e.g. mean arterial pressure $>90 \mathrm{mmHg}$ ) to maintain sufficient perfusion in the brain [23, 24]. Aggressive crystalloid resuscitation needs to be avoided [25]. These findings regarding fluid therapy should be taken in account for further improvement in the Finnish pre-hospital setting.

It took more time to perform the first CT scan in Navarra than in Southern Finland. Accordingly, it also increases the time to first surgical intervention. This may be because doctors attending trauma patients in Navarra may take more time in the evaluation of these patients. Furthermore, in hospitals of Navarra, CT scanner is located far from the resuscitation room and it takes time to reach there and perform imaging. It was shown that the location of the CT scanner in or near the trauma room, as opposed to a location at the Radiology Department, could also have a beneficial effect on outcome [26]. In the HUH trauma center the CT scanner is located next to the shock room. Changes should be done in hospital protocols and infrastructures to reduce these times in Navarra.

Elderly patients with severe head injury were treated more often in the ICU in southern Finland. It can be presumed that more severe cases tend to be admitted in the ICU. In this study, the Finnish trauma patients were more severely injured (as measured by ISS and NISS) than in Navarra. Furthermore, different indications for critical care admission may also explain the difference of ICU admission rate. The need for mechanical ventilation is one of the main reasons to admit patients to the ICU. In this study, more trauma patients who were on mechanical ventilation were documented in HTR than MTR-N. Also, patients in MTR-N were older compared to HTR. Treatment limitations in older patients with severe head injuries might be considered. In cases with hopeless prognosis treatment could be limited outside of the ICU.

Finnish patients also were transferred more frequently to another intermediate or low care somatic hospital ward than Navarra's patients. This is because in the HUH trauma unit, the number of ward beds is limited, and the goal is to step down injured patients to another hospital as soon as medically possible, which is when the need for ICU care is ceased and no more surgical procedures are planned.

Notable difference in GOS at discharge between the two registries (good recovery $78.5 \%$ in MTR-N and $8.2 \%$ in HTR) deserves consideration: GOS was originally developed to describe the level of neurological impairment after brain injury [27]. However, the Utstein template for uniform reporting of data following major trauma advises that the GOS should be analyzed at discharge and used in disability scaling also after such trauma as fractures and spinal cord injuries [1]. Thus, in HTR, a patient discharged at 2 weeks who has sustained no brain injury but a pelvic fracture obliging partial weight-bearing and help in daily activities for 8 weeks is considered severely disabled and earning a GOS 3 -though full recovery is expected after fracture healing. On the other hand, in some hospitals, the GOS is only used in its original sense: describing neurological impairment after brain injury. Thus, lack of consistency in data coding could be partly explaining the noted differences in GOS.

In addition, patients with more severe injuries require more time in recovering. As reflected by higher average NISS, and higher share of head AIS 5/6 and GCS $\leq 8$-patients, the more severely injured patients in HTR 
compared to MTR-N can be considered leading to worse GOS at discharge in HTR. Furthermore, it can be argued based on the outcome results that some of the patients with severe brain injury treated in HTR were survived as opposed to MTR-N. This might lead to a patient who is discharged alive but with severe disability (low GOS).

Low fall was the reason for severe brain injury in elderly in majority of the cases, especially in MTR-N. Older people make up a large and increasing percentage of the population. Evidence shows that specific programs for improving strength and balance can reduce the risk of falls by as much as 55\% [28]. It has also been demonstrated that falls prevention services are cost-effective and could make substantial savings [29]. The need of targeted programs preventing falls in elderly is emphasized by our study.

\section{Limitations of the study}

We acknowledge some limitations to our study. First, the Navarra registry and HTR have different data collection procedures. In Navarra patients are included into the database thanks to the collaboration of several users at different levels and a supervisor is responsible for checking adherence to the inclusion, exclusion and of the compliance of the Utstein style variables of each registered patient [17]. HTR contains data from one hospital coded by five dedicated trauma nurses. Although multiple plausibility controls are implemented, there is no source data verification to prevent entry errors. To minimize bias due to the limitations mentioned above, definitions were carefully checked, and data were transformed into comparable variables where necessary [18].

Second, this study utilizes data from two trauma registries, which collect data based on the Utstein criteria. This means that the threshold of including a patient to either of the registries is NISS (ISS) $\geq 16$. By definition, isolated head injuries with AIS 3 (NISS/ISS 9) are not included in our study, which is a limitation.

Third, HTR includes data solely from the only tertiary trauma centre in southern Finland. Since we excluded patients transferred from another hospital (due to missing initial status on admission), the prehospital setting undertriaged patients are missing from HTR. Even though the field triage of southern Finland pre-hospital system dictates all the suspected severe head trauma to be transferred to HUH trauma unit, it has previously been shown that delayed admission after TBI is common (35\%). However, no significant difference in 6-month mortality between TBI-patients arriving directly from the scene and transferred patients was noted, thus, this potential bias in outcome results is reduced [30]. Fourth, differences in trauma systems between Navarra and southern Finland explain some differences noted in results: the tendency to heavily centralize the treatment of severe head injuries, especially the ones benefitting from an emergency operation, in the HUH trauma unit is reflected in higher NISS, lower GCS, more frequent intubation and ICU utilization compared to Navarra. However, differences across the trauma systems and hospitals offer an opportunity to compare the different ways of treating trauma patients, which would not be possible within an existing system. Interestingly, more severe injuries seen in the HUH trauma unit did not lead to increase mortality.

Fifth and importantly, in patients with brain injury, 30-day hospital mortality significantly underestimates mortality rates [31] as mortality increases at least up to 6 months after the injury. HTR does not follow patients after discharge, while in MTR-N also discharged patients are followed up to 4 weeks. Thus, the tendency of HTR for early discharge (average 9 days) is a potential bias in the observed 30-day mortality (lowering the mortality rates in HTR). According to previously published data based on two large European registries (TR-DGU in Germany and TARN in England) about half of the in-hospital trauma deaths occur within the first $24 \mathrm{~h}$. However, $17-18 \%$ of the deaths occur between days $7-30$ and $4-5 \%$ of trauma-related deaths are recorded beyond 30 days [32].

Lack of follow-up and proper functional outcome measures after discharge prevents assessment of the long-term effects of severe trauma [33]. In severe brain injuries the emphasis should not be on only surviving the injury, but also on the quality of life for example 1 year after the injury.

\section{Conclusions}

The better adjusted outcome of elderly patients with severe brain injuries in southern Finland compared to Navarra could be due to higher rate of pre-hospital intubation and/or higher rate of admitting patients to ICU in southern Finland.

Instead of mere 30 days in-hospital mortality as an outcome method, knowledge on long-term outcomes and quality of life after TBI in aged population is needed to guide treatment decisions.

Increasing number of elderly patients with severe TBI associated with high mortality necessitate targeted programs preventing falls as well as uniformly accepted protocols in pre- and in-hospital management.

\section{Compliance with ethical standards}

Conflict of interest Bismil Ali, Tuomas Brinck, Lauri Handolin and Tomas Belzunegui declare that they have no conflict of interest.

Permission note (ethics) The study has been approved by the Ethics Committee of the Department of Health of the government of Navarra. The subjects gave their informed consent to the work. The investigation carried out was approved and evaluated by the National Agency 
for Quality and Accreditation of Spain, ANECA. This study has also been approved by the review board of the Helsinki University Hospital.

\section{References}

1. Ringdal KG, Coats TJ, Lefering R, Di Bartolomeo S, Steen PA, Røise O, Handolin L, Lossius HM. The Utstein template for uniform reporting of data following major trauma: a joint revision by SCANTEM, TARN, DGU-TR and RITG. Scand J Trauma Resusc Emerg Med 2008;16:7.

2. Grossman MD, Ofurum U, Stehly CD, Stoltzfus J. Long-term survival after major trauma in geriatric trauma patients: the glass is half full. J Trauma Acute Care Surg. 2012;72:1181-5.

3. Adams SD, Holcomb JB. Geriatric trauma. Curr Opin Crit Care. 2015;21(6):520-6.

4. WHO, Geneva: Switzerland; 2010. World Health Organisation. Definition of an older or elderly person. Accessed 12/11/2017.

5. Sammy I, Lecky F, Sutton A, Leaviss J, O'cathain A. Factors affecting mortality in older trauma patients-a systematic review and meta-analysis. Inj Int J Care Inj. 2016;47:1170-83.

6. Aitken LM, Burmeister E, Lang J, Chaboyer W, Richmond TS. Characteristics and outcomes of injured older adults after hospital admission. J Am Geriatr Soc. 2010;58(3):442-9.

7. Champion HR, Copes WS, Buyer D, Flanagan ME, Bain L, Sacco WJ. Major trauma in geriatric patients. Am J Public Health. 1989;79(9):1278-82.

8. Padrón-Monedero A, Damián J, Pilar Martin M, FernándezCuenca R. Mortality trends for accidental falls in older people in Spain, 2000-2015. BMC Geriatrics. 2017;17:276.

9. Alberdi F, García I, Atutxa L, Zabarte M. Epidemiology of severe trauma. Med intensiva. 2014;38(9):580-8.

10. Thompson HJ, McCormick WC, Kagan SH. Traumatic brain injury in older adults: epidemiology, outcomes and future implications. JAGS. 2006;54:1590-10.

11. Peck KA, Calvo RY, Sise CB, Johnson J, Yen JW, Sise MJ, Dunne CE, Badiee J, Shackford SR, Lobatz MA. Death after discharge: predictors of mortality in older brain injured patients. J Trauma Acute Care Surg. 2014;77:978-83.

12. Thompson HJ, Weir S, Rivara FP, Wang J, Sullivan SD, Salkever D, MacKenzie EJ. Utilization and costs of health care after geriatric traumatic brain injury. J Neurotrauma. 2012;29:1864-71.

13. Lefering R, Paffrath T, Linker R, Bouillon B, Neugebauer EA. German Society for Trauma Surgery. Head injury and outcome-what influence do concomitant injuries have? J Trauma. 2008;65:1036-43.

14. Ali Ali B, Lefering R, Fortun Moral M, Belzunegui Otano T. Epidemiological comparison between the Navarra Major Trauma Registry and the German Trauma Registry (TR-DGU ${ }^{\circledR}$ ). Scand J Trauma Resuscitation Emerg Med. 2017;25:107.

15. The ATSL Subcommittee, American College of Surgen's Committee on Trauma, and The International ATSL working group. Advanced Trauma Life Support (ATLS): the ninth edition. J Trauma Acute Care Surg. 2013;74:1363-6.

16. Thies K, Deakin CD, Lott C, Robinson D, Sabbe MB, Arafat R, Brattebo G, Lippert FK, Rommens PM, Voiglio EJ. The European trauma course-trauma teaching goes European. Resuscitation. 2013;85:19-20.
17. Ali Ali B, Lefering R, Otano Belzunegui T. Quality assessment of Major Trauma Registry of Navarra: completeness and correctness. Int J Injury Control Saf Promotion. https://doi.org/10.1080/17457 300.2018.1515229.

18. Heinänen M, Brinck T, Handolin L, Mattila VM, Söderlund T. Accuracy and coverage of diagnosis and procedural coding of severely injured patients in the finnish hospital discharge register: comparison to patient files and the Helsinki Trauma Registry. Scand J Surg. 2017;106(3):269-77.

19. Lefering R, Huber-Wagner S, Nienaber U, Maegele M, Bouillon B. Update of the trauma risk adjustment model of the TraumaRegister DGUTM: the Revised Injury Severity Classification, version II. Crit Care. 2014;18(5):476.

20. Giannoudis PV, Harwood PJ, Court-Brown C, Pape HC. Severe and multiple trauma in older patients; incidence and mortality. Injury. 2009;40(4):362-7.

21. Wang HE, Cook LJ, Chang CC, Yealy DM, Lave JR. Outcomes after out-of hospital endotracheal intubation errors. Resuscitation. 2009;80:50-5.

22. Cranshaw J, Nolan J. Airway management after major trauma. Contin Educ Anaesth Crit Care Pain. 2006;6:124-27.

23. Haddad SH, Arabi YM. Critical care management of severe traumatic brain injury in adults. Scand J Trauma Resusc Emerg Med. 2012;20:12.

24. Kinoshita K. Traumatic brain injury: Pathophysiology for neurocritical care. J Intensive Care. 2016;4:29.

25. Chatrath V, Khetarpal R, Ahuja J. Fluid management in patients with trauma: restrictive versus liberal approach. J Anaesthesiol Clin Pharmacol. 2015;31(3):308-16.

26. Huber-Wagner S, Mand C, Ruchholtz S, Kuhne CA, Holzapfel K, Kanz KG, Griensven MV, Biberthaler P, Lefering R. the Trauma Register DGU. Effect of the localization of the CT scanner during trauma resuscitation on survival-a retrospective, multicentre study. Lancet. 2009;373:1455-61.

27. Jennett B, Bond M. Assessment of outcome after severe brain damage: a practical scale. The Lancet. 1975;305:480-4.

28. Logan PA, Coupland CA, Gladman JR, Sahota O, Stoner-Hobbs V, Robertson K, Tomlinson V, Ward M, Sach T, Avery AJ. Community falls prevention for people who call an emergency ambulance after a fall: randomised controlled trial. BMJ. 2010;340:c2102-10.

29. Centre for Clinical Practice at NICE (UK) Falls: assessment and prevention of falls in older people. In: NICE Clinical Guidelines, No. 161. London: National Institute for Health and Care Excellence;2013.

30. Raj R, Siironen J, Kivisaari R, Kuisma M, Brinck T, Lappalainen J, Skrifvars MB. Factors correlating with delayed trauma center admission following traumatic brain injury. Scand J Trauma Emerg Surg. 2013;21:67.

31. Fleischman RJ, Adams AL, Hedges JR, Ma OJ, Mullins RJ, Newgard CD. The optimum follow-up period for assessing mortality outcomes in injured elders. J Am Geriatr Soc. 2010;58(10):1843-9.

32. Lefering R, Paffrath T, Bouamra O, Coats TJ, Woodford M, Jenks T, Wafaisade A, Nienaber U, Lecky F. Epidemiology of in-hospital trauma deaths. Europ J Trauma Emerg Med. 2012;38:3-9.

33. Hansen L, Shaheen A, Crandall M. Outpatient follow-up after traumatic injury: challenges and opportunities. J Emerg Trauma Shock. 2014;7(4):256-60. 